\title{
Novel microfluidic technologies for portable diagnostics systems
}

\author{
Abraham Lee \\ From Immunodiagnosis of Tuberculosis: New Questions, New Tools \\ Virginia, VA, USA. 21-23 September 2008
}

Novel microfluidic technologies being developed for portable diagnostic systems have potential for use in TB serodiagnosis or as platforms for cell and biomolecular assays. Using microfluidics, with its miniaturized channels and reservoirs for portable devices, offers many advantages, including: high surface-to-volume ratio; a low Reynolds number; increased speed of reaction; reduced cost of reagents; decreased cost of power consumption; precise mixing/dosage and heating. Its integration capability offers advantages as well, such as low manufacturing cost and multiplex capability, i.e. an increased number of parameters can be monitored per sample.

Two microfluidic platforms may be helpful in protein microarray analysis. Microfluidic electrical sensing platforms are capable of detecting antigen/antibody binding in real time. The development of this platform will allow for faster detection of certain diseases with a patient's sample compared to standard techniques such as ELISA. This platform requires microchannel-sealed microarrays with electrical detection capability. By measuring electrical charges, the scientists can determine protein-binding activity. Additionally, microfluidic colorimetric platforms are being developed using acoustic micromixing instead of fluorescence scanning. The process uses a scanner that detects an enzyme-based change of color that allows measurement of the proteinbinding event. Our team is developing these platforms using components that have very small energy requirements, which means the device may not require batteries to operate.

A 'lab-in-a-droplet' technique using digital microfluidics rather than using microarrays, which produce a static substrate, diffuses samples into as many as millions of droplets, presenting a three-dimensional picoliter reactor array. This process has several advantages including: rapid mixing, homogeneous reactions, precise control of volumes and concentrations, and an ability to mimic cellular reactions. Our team is using this technique in the development of lipoplexes and to encapsulate a cell in a single droplet. Lastly, a platform is under development for cell sorting using electrodes to track cells in order to determine what function the cell will take on.

In addition to its value in the diagnosis of $\mathrm{TB}$, microfluidics technology has several other applications, including: broader distribution of new assays to biologic labs, rapid analyses in the field for epidemiologic studies, and the ability to study hybridization of molecules on microarrays. For example, Lab-on-a-chip technologies are an excellent vehicle for integrating diagnostic and therapeutic functions. Digital (droplet) microfluidics enables high throughput cellular and molecular assays and cell sorting technologies enable more practical cellular analyses and therapies.

Published: 17 December 2010

doi:10.1186/1753-6561-4-S3-O19

Cite this article as: Lee: Novel microfluidic technologies for portable diagnostics systems. BMC Proceedings 2010 4(Suppl 3):O19. 